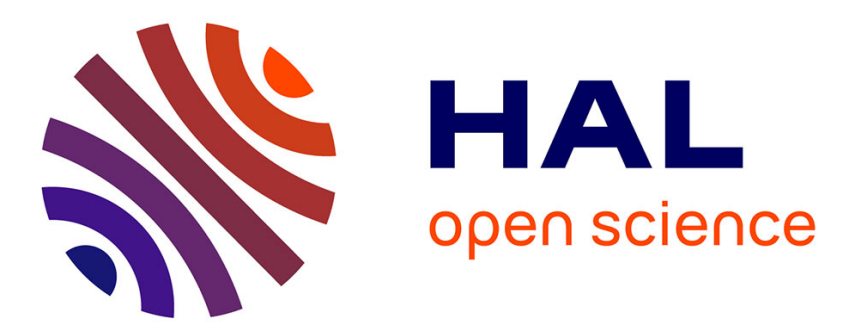

\title{
Prediction of self-heating measurements under proportional and non-proportional multiaxial cyclic loadings
}

Martin Poncelet, Cédric Doudard, Sylvain Calloch, François Hild, Bastien Weber, André Galtier

\section{To cite this version:}

Martin Poncelet, Cédric Doudard, Sylvain Calloch, François Hild, Bastien Weber, et al.. Prediction of self-heating measurements under proportional and non-proportional multiaxial cyclic loadings. Comptes Rendus Mécanique, 2007, 335, pp.81-86. 10.1016/j.crme.2007.01.002 . hal-00199382

\section{HAL Id: hal-00199382 https://hal.science/hal-00199382}

Submitted on 18 Dec 2007

HAL is a multi-disciplinary open access archive for the deposit and dissemination of scientific research documents, whether they are published or not. The documents may come from teaching and research institutions in France or abroad, or from public or private research centers.
L'archive ouverte pluridisciplinaire HAL, est destinée au dépôt et à la diffusion de documents scientifiques de niveau recherche, publiés ou non, émanant des établissements d'enseignement et de recherche français ou étrangers, des laboratoires publics ou privés. 


\title{
Prediction of self-heating measurements under proportional and non-proportional multiaxial cyclic loadings
}

\author{
Martin Poncelet ${ }^{\mathrm{a}}$, Cédric Doudard ${ }^{\mathrm{b}}$, Sylvain Calloch ${ }^{\mathrm{b}}$, François Hild ${ }^{\mathrm{a}}$, Bastien Weber ${ }^{\mathrm{c}}$ \\ André Galtier ${ }^{\mathrm{C}}$ \\ ${ }^{a}$ LMT-Cachan, ENS de Cachan, CNRS-UMR 8535, Université Paris 6, 61 av. du Président Wilson, F-94235 Cachan \\ cedex, France \\ b Laboratoire de Mécanique des Structures Navales, ENSIETA, 2 rue François Verny, F-29806 Brest cedex 9, France \\ ${ }^{\mathrm{c}}$ ARCELOR Research, voie romaine BP 30320, F-57283 Maizières-lès-Metz cedex, France \\ Received $* * * *$; \\ Presented by
}

\begin{abstract}
Self-heating measurements under cyclic loadings allow for fast estimations of fatigue properties. These tests are performed under tension-torsion loadings on a medium-carbon steel and a model accounting for heterogeneities is proposed to analyse heat transfer results. Both proportional and non-proportional loading paths are predicted. To cite this article:

\section{Résumé}

Prévision d'essais d'auto-échauffement sous chargements multiaxiaux hétérogènes. Les essais d'autoéchauffement permettant une estimation rapide des caractéristiques de fatigue. Ils sont réalisés ici sur un acier C45 sous chargements de traction-torsion. Un modèle prenant en compte l'hétérogénéité de la dissipation est proposé. Il permet de rendre compte des mesures thermiques sous trajets proportionnel et non-proportionnel. Pour citer cet article :
\end{abstract}

Key words: Fatigue; Microplasticity ; Poisson point process ; Heterogeneous stress ; Dissipation

Mots-clés : Fatigue; Microplasticité; Processus ponctuel de Poisson; Contraintes hétérogènes ; Dissipation

\footnotetext{
Email addresses: poncelet@lmt.ens-cachan.fr (Martin Poncelet), cedric.doudard@ensieta.fr (Cédric Doudard), sylvain.calloch@ensieta.fr (Sylvain Calloch), hild@lmt.ens-cachan.fr (François Hild), weber@arcelor.com (Bastien Weber), galtier@arcelor.com (André Galtier).
} 


\section{Introduction}

For several years, different methods of rapid estimation of mean fatigue limits based on temperature measurements have been developed. These tests consist in measuring the change of the specimen temperature under (usually) uniaxial cyclic loadings with thermocouples $[1,2]$ or thermography [3,4]. The amplitude of loading is step-wise constant and risen once the temperature is stabilised. The steady-state temperature is plotted as a function of the loading amplitude as shown in Figure 2. For some materials (e.g., steel or cast iron), a first part of the curve shows virtually no change in temperature, whereas in the second part the temperature rises significantly with the stress amplitude. A correlation between the mean fatigue limit and the stress level leading to the temperature increase has been empirically proposed $[3,4,2]$. Currently, models $[5,6]$ based on microplasticity are developed to describe this result. To take into account the progressive appearance of microplasticity two approaches are proposed. In the first one [5], the authors consider that the volume fraction of microplastic inclusion depends on the stress amplitude. In the second one [2], a probabilistic approach is used. The last model enables one to relate the fatigue behaviour with temperature measurements.

The investigation of multiaxial fatigue needs time-consuming experimental databases (e.g., stair case tests for each loading direction). Applying temperature measurements to multiaxial fatigue is thus very interesting as shown with biaxial loadings [7]. However, it is seldom performed. It is proposed to carry out and analyse a more common type of loading such as tension-torsion. First, a probabilistic two-scale model is extended to multiaxial heterogeneous loadings. Then it is applied and finally validated with proportional and non-proportional loading results.

\section{Modeling}

\subsection{Model basis}

Gradual rise of heat (and thus temperature) is supposed to be a consequence of a random onset of microplasticity, which has been observed on low carbon steel [8]. The distribution of active grains (later called "sites") in relation to their surrounding explain the "probabilistic" feature, which is modeled by a Poisson Point Process. The probability of finding $k$ sites in a domain $\Omega$ of volume $V$ reads

$$
P_{k}(V)=\frac{(\lambda V)^{k}}{k !} \exp (-\lambda V),
$$

where $\lambda V$ is the mean number of sites under a given load amplitude and $\lambda$ the "intensity" of the Poisson Point Process, depending on an equivalent stress $\Sigma_{a}$ (later called "Equivalent Activation Stress") of the load amplitude and two material parameters $m$ and $V_{0} S_{0}^{m}$

$$
\lambda=\frac{1}{V_{0}}\left(\frac{\Sigma_{a}}{S_{0}}\right)^{m}
$$

where $\Sigma_{a}$ is supposed to be equal to Von Mises equivalent stress amplitude $\Sigma_{0}^{e q}$. Experimental validation was performed for a Dual Phase steel [7]. The stress tensor in the plastic inclusion $\boldsymbol{\sigma}$ is related to the macroscopic stress tensor $\boldsymbol{\Sigma}$ and the corresponding strain tensor $\boldsymbol{\epsilon}^{p}$ by [9]

$$
\boldsymbol{\sigma}=\boldsymbol{\Sigma}-2 \mu(1-\beta) \boldsymbol{\epsilon}^{p},
$$

where $\mu$ denotes the shear modulus and $\beta$ a constant related to the Poisson's ratio of the material.

The magnitude of the intrinsic dissipated energy $D_{i n c}\left(\Sigma_{0}^{e q}, \sigma_{y}\right)$ in a site over a loading cycle is calculated for a given value of the yield stress $\sigma_{y}$ and a Von Mises equivalent stress amplitude $\Sigma_{0}^{e q}$. A Von Mises 
equivalent stress for the plasticity criterion associated with a linear kinematic hardening is assumed. For a uniform proportional loading, $D_{i n c}\left(\Sigma_{0}^{e q}, \sigma_{y}\right)$ is given by

$$
D_{i n c}^{\text {prop }}\left(\Sigma_{0}^{e q}, \sigma_{y}\right)=\frac{4 \sigma_{y}^{u}}{h}\left\langle\Sigma_{0}^{e q}-\sigma_{y}^{u}\right\rangle
$$

where $h$ is the hardening modulus. The total dissipated energy is then obtained by integration over the whole population of sites $[6]$

$$
D\left(\Sigma_{0}^{e q}\right)=\int_{0}^{\Sigma_{0}^{e q}} D_{i n c}\left(\Sigma_{0}^{e q}, \sigma\right) \frac{d \lambda}{d \sigma} d \sigma .
$$

For a heterogeneous stress field over a domain $\Omega$ of volume $V$, the global (mean) dissipated energy $\Delta$ is expressed as

$$
\Delta=\frac{1}{V} \int_{\Omega} D\left(\Sigma_{0}^{e q}\right) d V
$$

In the following, it is assumed that the temperature is uniform. This hypothesis will be discussed for the experimental application. The mean dissipation $\Delta$ is then introduced in the following heat conduction equation

$$
\dot{\Theta}+\frac{\Theta}{\tau_{e q}}=\frac{f_{r} \Delta}{\rho C},
$$

where $\tau_{e q}$ is a characteristic time depending on the heat transfer boundary condition [11], $\rho$ the mass density, $C$ the specific heat, $f_{r}$ the load frequency and $\Theta$ the mean temperature. There is no need to add a thermoelastic term, which is vanishing over one cycle because only mean steady-state temperatures are sought. The mean (uniform) steady-state temperature $\bar{\Theta}$ can then be calculated.

For proportional loadings, $\Delta$ is simplified as

$$
\Delta=\frac{4 m V_{0}}{h(m+1)(m+2)} \frac{H_{m+2}\left(\Sigma_{0 M}^{e q}\right)^{m+2}}{V_{0} S_{0}^{m}}=\frac{4 m V_{0}}{h(m+1)(m+2)} \frac{\left(\Sigma_{\text {eff diss }}\right)^{m+2}}{V_{0} S_{0}^{m}},
$$

where $\Sigma_{0 M}^{e q}=\max _{\Omega}\left(\Sigma_{0}^{e q}\right), \Sigma_{\text {eff diss }}$ the effective dissipative stress and $H_{m+2}$ the heterogeneity stress factor [10] given by

$$
H_{m+2}=\frac{1}{V} \int_{\Omega}\left(\frac{\Sigma_{0}^{e q}}{\Sigma_{0 M}^{e q}}\right)^{m+2} d V
$$

For non-proportional loadings, it is no longer possible to simplify $\Delta$ because of the expression of $D\left(\Sigma_{0}^{e q}\right)$. $\Delta$ is then calculated with the same hypotheses, but using a numerical integration software. However, it is possible to define a non-proportional loading factor

$$
G_{m+2}=\Delta / \Delta_{0 M},
$$

where $\Delta_{0 M}$ is the mean dissipated energy for a proportional and uniform loading and a Von Mises stress amplitude $\Sigma_{0 M}^{e q}$ defined by

$$
\Delta_{0 M}=\frac{4 m V_{0}}{h(m+1)(m+2)} \frac{\left(\Sigma_{0 M}^{e q}\right)^{m+2}}{V_{0} S_{0}^{m}} .
$$

It is thus possible to extend the concept of effective stress for non-proportional loading as follows

$$
\Sigma_{\text {eff diss }}=G_{m+2}^{1 /(m+2)} \Sigma_{0 M}^{e q},
$$

where $G_{m+2}=H_{m+2}$ for proportional loadings. The steady-state temperature $\bar{\Theta}$ reads in any case

$$
\bar{\Theta}=\eta \frac{m}{(m+1)(m+2)} \frac{\left(\Sigma_{\text {eff diss }}\right)^{m+2}}{V_{0} S_{0}^{m}}
$$


with $\eta=4 f_{r} \tau_{e q} / h \rho C$. Equation (13) shows that the thermal behaviour depends only on three parameters and the effective dissipative stress. In the following section, the previous analysis is applied to and validated on a medium carbon steel C45 (SAE45).

\section{Validation of tension-torsion results}

\subsection{Experimental configuration}

A tubular specimen with 2 flat sections per head [12] is used for tension-torsion loadings. The ratio thickness/mean radius of the gauge section is equal to 0.24 (external and internal radius are respectively $R_{e}=7 \mathrm{~mm}$ and $R_{i}=5.5 \mathrm{~mm}$ ). All samples are machined from the same bar of medium carbon steel C45 (SAE45), which is, as far as elastic and thermal parameters are concerned, homogeneous and isotropic. Experiments are performed using a tension-torsion servohydraulic-testing machine. Differential temperature measurements are obtained by using 2 thermocouples, one in contact with the centre of the external surface of the gauge zone and the other on the grips of the actuator. At a loading frequency of $5 \mathrm{~Hz}$, 3,000 cycles are needed for each step to reach steady state conditions. The macroscopic stress tensor that depends on the radius $r$, is expressed as

$$
\boldsymbol{\Sigma}(r)=\left[\begin{array}{cll}
\Sigma_{11} & \Sigma_{12} & 0 \\
\Sigma_{12} & 0 & 0 \\
0 & 0 & 0
\end{array}\right], \quad \text { with } \quad \begin{gathered}
\Sigma_{11}=\Sigma_{\max } \cos \left(2 \pi f_{r} t\right) \\
\quad \Sigma_{12}=\tau_{\max } \frac{r}{R e} \cos \left(2 \pi f_{r} t+\delta\right)
\end{gathered}
$$

where $f_{r}$ is the loading frequency, $\Sigma_{\max }$ and $\tau_{\max }$ the tensile and shear stress amplitudes and $\delta$ the phase difference between shear and normal stresses.

A pure tension and a pure torsion loading path are first performed on two different samples. On a third one, a non-proportional path, later called "out-of-phase" is carried out, corresponding to a Von Mises circle in the tension-torsion stress space for a point of the gauge section at mean radius. One must note that for other points, the loading path is still an ellipse, but with the major to minor axis ratio different from $\tau_{\max } / \Sigma_{\max }=\sqrt{3}$.

\subsection{Validation of the uniformity assumption of steady-state temperature}

The heat transfer boundary conditions are a constant and uniform temperature on the interface sample/grip $\left(\theta_{\text {grips }}=0\right)$ and a convection condition $\left(\frac{\partial \theta}{\partial r}\right) \pm h_{1} \theta=0$ on respectively internal and external faces of the tube, where the value of the heat transfer coefficient $h_{1}$ is taken from the literature [11]. Grips are considered to be "far" from the gauge section, so that the steady-state temperature of the gauge section $\bar{\theta}$ is considered as a simple function of the radius $r$. The heat equation to solve reads

$$
\dot{\bar{\theta}}-\frac{k}{\rho C} \frac{\partial}{r \partial r}\left(r \frac{\partial \bar{\theta}}{\partial r}\right)=\frac{f_{r} \Delta(r)}{\rho C},
$$

where $k$ is the thermal conductivity. Figure 1 shows in pure torsion that $\theta$ is nearly uniform (i.e., variation less than $0.02 \%$ ) despite a high heterogeneity of dissipation, which is experimentally checked by measuring temperatures on both external and internal surfaces. The steady-state temperature is thus uniform and Eq. (13) applies. 


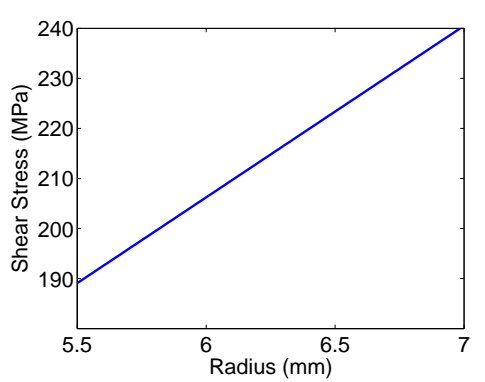

(a)

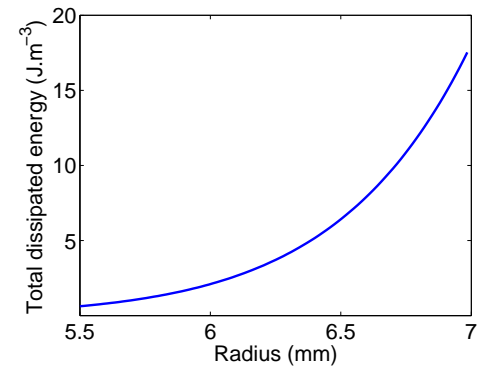

(b)

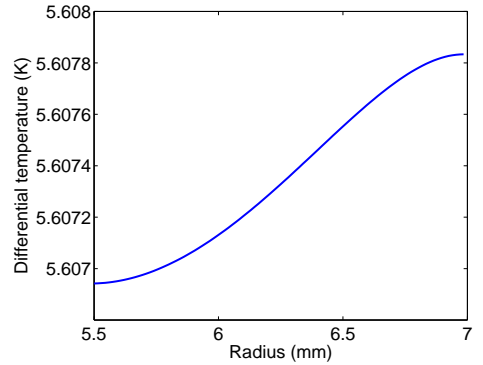

(c)

Figure 1. Change of shear stress (a), total dissipated energy (b) and temperature (c) as functions of radius $\left(k=50 \mathrm{Wm}^{-1} \mathrm{~K}^{-1}, \rho=7,900 \mathrm{kgm}^{-3}, C=500 \mathrm{Jkg}^{-1} \mathrm{~K}^{-1}, \tau_{e q}=70 \mathrm{~s}\right)$.

\subsection{Evaluation of the effective dissipative stress}

In proportional tension-torsion, $G_{m+2}$ reads

$$
G_{m+2}=\frac{2}{R_{e}^{2}-R_{i}^{2}} \int_{R_{i}}^{R_{e}}\left(\frac{\sigma_{\max }^{2}+3\left(\frac{r \tau_{\max }}{R_{e}}\right)^{2}}{\sigma_{\max }^{2}+3 \tau_{\max }^{2}}\right)^{\frac{m+2}{2}} r d r
$$

and for the non-proportional loading, $G_{m+2}$ is numerically calculated. As shown in Table 1 for the present geometry and material parameters, values of $G_{m+2}$ for heterogeneous out-of-phase loading path (hypothesis of tension-torsion on thick-walled specimen) and uniform out-of-phase loading path (hypothesis of thin-walled specimen) influence the result. In the case of out-of-phase loading path, values of $G_{m+2}$ greater than one are due to higher intrinsic dissipated energy than for proportional loading.

Table 1

Values of $H_{m+2}$ and $G_{m+2}$ for different loading path when $R_{e}=7 \mathrm{~mm}, R_{e}=5.5 \mathrm{~mm}, m=12$.

\begin{tabular}{|c||c|c|c|c|}
\hline & Tension & Torsion & Heterogeneous out-of-phase & Uniform out-of-phase \\
\hline \hline$H_{m+2}$ & 1 & 0.32 & 0.36 & 1 \\
\hline$G_{m+2}$ & 1 & 0.32 & 7.29 & 7.24 \\
\hline
\end{tabular}

The experimental analysis consists in identifying the parameter $m$ (e.g., using the steady-state temperature measurements in tension [2], see Figure 2(a)) and then calculating the different heterogeneity factors to plot the other paths as shown in Figure 2(b). To show the influence of the factor $G_{m+2}$, experimental results are directly plotted in Figure 2(a) as functions of Von Mises equivalent stress amplitude. Figure 2(b) shows that the effective stress allows one to collapse all the experimental data onto a single curve.

\section{Summary}

Self-heating measurements under different proportional and non-proportional cyclic loadings have been performed in tension-torsion. Plotting results as functions of Von Mises equivalent stress amplitude is not the adequate method (Figure 2(a)). The stress heterogeneity effect induced by the probabilistic treatment of the experiments needs to be accounted for. Last, by using only one loading path results (e.g., tension) to identify the parameters, it is possible to calculate a heterogeneity factor that allows for the prediction 


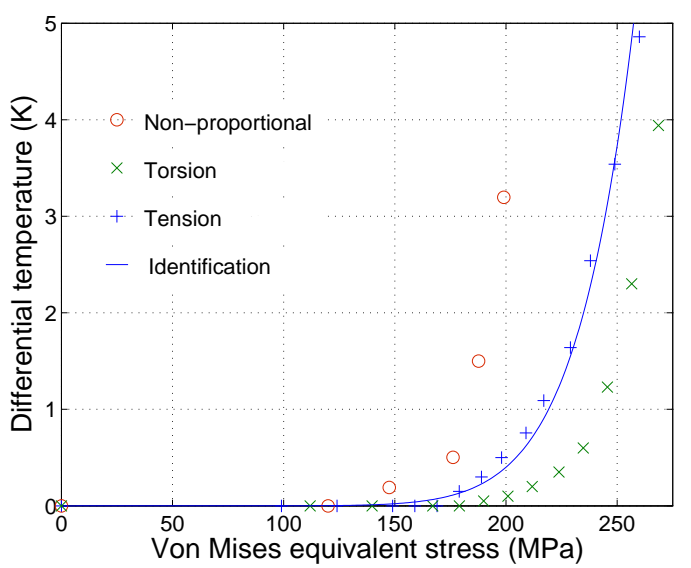

(a)

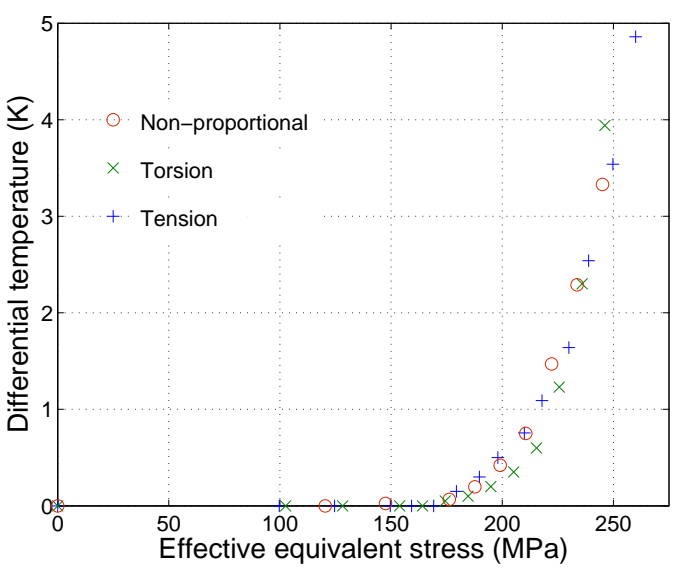

(b)

Figure 2. Steady-state temperature for different loading paths: tension $(+)$, torsion $(\times)$ and non-proportional $(\circ)$ as functions of Von Mises equivalent stress amplitude (a) and effective stress amplitude (b).

of all other tested loading paths as shown in Figure 2(b). The next step is the fatigue prediction under multiaxial loadings and its validation.

\section{References}

[1] A. Galtier, O. Bouaziz and A. Lambert, Influence de la microstructure des aciers sur leurs propriétés mécaniques, Méc. Ind., 3:457-462, 2002.

[2] C. Doudard, S. Calloch, F. Hild, P. Cugy and A. Galtier, A probabilistic two-scale model for high cycle fatigue life predictions, Fat. Fract. Engng. Mater. Struct., 28:279-288, 2005.

[3] J.-C. Krapez, D. Pacou and C. Bertin, Application of lock-in thermography to rapid evaluation of fatigue limit in metals, Proceedings of the $5^{\text {th }}$ AITA, Venezia (Italy), 1999.

[4] G. La Rosa and A. Risitano, Thermographic methodology for rapid determination of the fatigue limit of materials and mechanical components, Int. J. Fatigue, 22:65-73, 2000.

[5] E. Charkaluk, A. Constantinescu, Estimation of the mesoscopic thermoplastic dissipation in High-Cycle Fatigue, C.R. Mecanique, 334:373-379, 2006.

[6] C. Doudard, S. Calloch, F. Hild, P. Cugy and A. Galtier, Identification of the scatter in high cycle fatigue from temperature measurements, C.R. Mecanique, 332:795-801, 2004.

[7] C. Doudard, M. Poncelet, S. Calloch, C. Boue, F. Hild and A. Galtier, Determination of an HCF criterion by thermal measurements under biaxial cyclic loading, Int. J. Fat., in press, 2006.

[8] P. Cugy and A. Galtier, Microplasticity and temperature increase in low carbon steel, Proceedings of the $7^{\text {th }}$ Int. Fat. Conf., Stockholm (Sweden), 2002.

[9] M. Berveiller and A. Zaoui, An extension of the self-consistent scheme to plastically flowing polycrystals, J. Mech. Phys. Solids, 26:325-344, 1979.

[10] F. Hild, R. Billardon and D. Marquis, Héétérogénéité des contraintes et rupture des matériaux fragiles, C.R. Acad. Sci. Paris, II 315:1293-1298, 1992.

[11] A. Chrysochoos and H. Louche, An infrared image processing to analyse the calorific effects accompanying strain localisation, Int. J. Eng. Sc., 38:1759-1788, 2000.

[12] J. Lemaitre and J.-L. Chaboche Mécanique des matériaux solides, Dunod, Paris, 1988, p. 90. 